\title{
New Simplified Formulas for ELF Subsurface-to-Subsurface Propagation
}

\author{
PETER R. BANNISTER, MEMBER, IEEE
}

(Invited Paper)

\begin{abstract}
New formulas for the electric and magnetic fields produced by the four elementary dipole antennas have been developed for the subsurface-to-subsurface propagation case. The formulas are of rather simple, but useful, form and are completely general (i.e., the air can easily be replaced by the sea bottom). They are valid at any frequency and at any range beyond a certain minimum distance for the flat-earth case. They are especially applicable at extremely low frequency (ELF) for the air/sea and sea/ seabed propagatipn cases. The main restrictions on these formulas are 1) the square of the index of refraction is greater than or equal to 10 and 2) the horizontal separation is greater than or equal to 3 times the sum of the depths of burial of the transmitting and receiving point sources. With these new formulas, computer eraluation can be reduced to fractions of a minute, compared with hours for the complete numerical evaluation of the exact Sommerfeld integrals. The formulas also exhibit an interference pattern set up under certain close-range conditions. The three wayes (direct, modified mirror image, and lateral) may interfere, either constructively or destructively, yith each other.
\end{abstract}

\section{INTRODUCTION}

$\mathbf{I}^{\mathrm{N}}$ $\mathrm{N}$ THE PAST, many investigators have erroneously believed that the field-strength equations tabulated in Kraichman $[1$, ch. 3] are only valid when the conduction currents in the water or earth are much greater than the displacement currents (i.e., $\sigma_{1} \gg \omega \epsilon_{1}$ ). Indeed, as long as $\left|n^{2}\right| \gg 1$, the displacement currents can be included simply by replacing $\sigma_{1}$ with $\sigma_{1}+i \omega \epsilon_{1}$ in the field-strength equations. Thus Kraichman's tabulated results are considerably more general than they are stated to be. (The index of refraction $n$ is equal to $\gamma_{1} / \gamma_{0}$, where $\left.\gamma_{1}\left(=\sqrt{i \omega \mu_{0}\left(\sigma_{1}+i \omega \epsilon_{1}\right.}\right)\right)$ is the propagation constant in the water or earth, and $\gamma_{0}\left(=i \omega \sqrt{\mu_{0} / \epsilon_{0}}\right)$ is the propagation constant in the air.)

It is the purpose of this article to extend the range of validity of Kraichman's results and present new formulas for horizontal electric dipole (HED), horizontal magnetic dipole HMD), vertical electric dipole (VED), and vertical magnetic dipole (VMD) subsurface-to-subsurface propagation. These formulas have been obtained complete from previously derived results. The main restrictions on their use are 1) the square of the index of refraction $\left(\left|n^{2}\right|\right)$ is greater than or equal to 10 and 2) the horizontal separation is greater than or equal to three times the sum of the depths of burial of the transmitting and receiving point șources. An additional restriction must also be applied to the lateral-wave components. The quantity $\left|\gamma_{1} \rho^{2} /(z+h)\right|$ must be greater than or equal

Manuscript received October 18, 1983; revised April 2, 1984.

The author is with the Submarine Electromagnetic Systems Department, Naval Underwater Systems Center, New London, CT 06320. to $4 c_{1}$, where $c_{1}=3,6,9,15$, or 25 , depending on the particular field-strength component.

In this article, the four dipole antennas (VED, VMD, HED, and HMD) are situated at depth $h(h \geqslant 0)$ with respect to a cylindrical coordinate system $(\rho, \phi, z)$ and are assumed to carry a constant current $l$. The axes of the VED and HED (of dipole moment $p$ ) are oriented in the $z$ and $x$ directions, respectively, and the axes of the VMD and HMD (of dipole moment $m$ ) are oriented in the $z$ and $y$ directions, respectively. The earth or water occupies the lower half-space (medium $1, z>0$ ) while the air occupies the upper half-space (medium $0, z<0$ ). The magnetic permeability of the earth is assumed to equal $\mu_{0}$, the permeability of free space. The International System (SI) of units is employed and the suppressed time factor of $\exp (i \omega t)$ is assumed.

In three recent papers, Wu and King [2] - [4] have derived new simple formulas for the six field components generated by a horizontal electric dipole in a half-space of water or earth near its boundary with air. They indicate that their formulas are valid for $\rho^{2} \gg(z+h)^{2}$ and $\left|n^{2}\right| \gg 1$. On examining their results, the author has discovered that they could have been obtained almost by inspection from the previously derived results of Wait [5], Weaver [6], Bannister [7], and Bannister and Hart [8] (most of which are summarized in Kraichman [1]). Also, five out of the six field component expressions derived by $\mathrm{Wu}$ and $\mathrm{King}$ involve the unphysical distance $z+h+\rho$. Their use of this unphysical distance leads to errors in the range where the various waves interfere, either constructively or destructively, with each other. These errors are discussed in more detail in the Appendix.

\section{DERIVATION PROCEDURE}

As we shall soon see, the unphysical distance $z+h+\rho$ can be avoided. When both the transmitting and receiving dipoles are located below the earth's surface ( $h$ and $z \geqslant 0$ ), the HED hertz vector potential $\Pi_{x}$ may be expressed as (Wait [5]):

$$
\Pi_{x}=\frac{p}{4 \pi\left(\sigma_{1}+i \omega \epsilon_{1}\right)}\left[P_{0}-P_{1}+I\right]
$$

where

$$
P_{0}=\frac{e^{-\gamma_{1} R_{0}}}{R_{0}}
$$


is the direct-wave contribution and

$$
P_{1}=\frac{e^{-\gamma_{1} R_{1}}}{R_{1}}
$$

is the mirror-image contribution. The remaining term is

$$
\begin{aligned}
I & =2 \int_{0}^{\infty} \frac{e^{-u_{1}(z+h)}}{u_{1}+u_{0}} J_{0}(\lambda \rho) \lambda d \lambda \\
& =\frac{2}{\left(\gamma_{1}{ }^{2}-\gamma_{0}{ }^{2}\right)} \int_{0}^{\infty}\left(u_{1}-u_{0}\right) e^{-u_{1}(z+h)} J_{0}(\lambda \rho) \lambda d \lambda
\end{aligned}
$$

where $R_{0}{ }^{2}=\rho^{2}+(z-h)^{2}, R_{1}{ }^{2}=\rho^{2}+(z+h)^{2}, u_{0}{ }^{2}=$ $\lambda^{2}+\gamma_{0}{ }^{2}$, and $u_{1}{ }^{2}=\lambda^{2}+\gamma_{1}{ }^{2}$. When the measurements distance is much less than a free-space wavelength, (1) reduces to

$$
\begin{aligned}
\Pi_{x} \approx & \frac{p}{4 \pi\left(\sigma_{1}+i \omega \epsilon_{1}\right)}\left[P_{0}-P_{1}\right. \\
& \left.+\frac{2}{\left(\gamma_{1}{ }^{2}-\gamma_{0}{ }^{2}\right)} \int_{0}^{\infty}\left(u_{1}-\lambda\right) e^{-u_{1}(z+h)} J_{0}(\lambda \rho) \lambda d \lambda\right] .
\end{aligned}
$$

Wait [5] has shown that this equation is equivalent to

$$
\begin{aligned}
\Pi_{x} \approx & \frac{p}{4 \pi\left(\sigma_{1}+i \omega \epsilon_{1}\right)}\left[P_{0}-P_{1}\right. \\
& \left.+\frac{2}{\left(\gamma_{1}{ }^{2}-\gamma_{0}{ }^{2}\right)}\left(\frac{\partial^{2} P_{1}}{\partial z^{2}}-\frac{\partial^{3} N}{\partial z^{3}}+\gamma_{1}{ }^{2} \frac{\partial N}{\partial z}\right)\right]
\end{aligned}
$$

where

$$
N=I_{0}\left\{\frac{\gamma_{1}}{2}\left[R_{1}-(z+h)\right]\right\} K_{0}\left\{\frac{\gamma_{1}}{2}\left[R_{1}+(z+h)\right]\right\}
$$

and that, when $\left|\gamma_{1} \rho\right| \gg 1$ and $\rho \gg(z+h)$

$$
N \approx \frac{e^{-\gamma_{1}(z+h)}}{\gamma_{1} \rho}
$$

To quote Wait [5], "The manner in which the exponential factor exp $\left[-\gamma_{1}(z+h)\right]$ occurs is rather interesting. It is only in the integral $N$ that this factor emerges."

If we substitute (8) into (5) and take the indicated derivatives, (5) reduces to

$$
\begin{aligned}
\Pi_{x} \approx & \frac{p}{4 \pi\left(\sigma_{1}+i \omega \epsilon_{1}\right)}\left\{\frac{e^{-\gamma_{1} R_{0}}}{R_{0}}+\frac{2 e^{-\gamma_{1}(z+h)}}{\left(\gamma_{1}{ }^{2}-\gamma_{0}{ }^{2}\right) \rho^{3}}\right. \\
& -\frac{e^{-\gamma_{1} R_{1}}}{R_{1}}-\frac{2 e^{-\gamma_{1} R_{1}}}{\left(\gamma_{1}{ }^{2}-\gamma_{0}{ }^{2}\right) R_{1}{ }^{3}}\left[\left(1-3 \sin ^{2} \psi_{1}\right)\right. \\
& \left.\left.\cdot\left(1+\gamma_{1} R_{1}\right)-\gamma_{1}{ }^{2} R_{1}{ }^{2} \sin ^{2} \psi_{1}\right]\right\}
\end{aligned}
$$

where $\sin \psi_{1}=(z+h) / R_{1}$. For $\rho^{2} \gg(z+h)^{2}$, we can set $R_{0}$ and $R_{1}$ equal to $\rho$ everywhere except in the exponents. Thus

$$
\begin{aligned}
\Pi_{x} \approx & \frac{p}{4 \pi\left(\sigma_{1}+i \omega \epsilon_{1}\right)}\left\{\frac{e^{-\gamma_{1} R_{0}}}{\rho}+\frac{2 e^{-\gamma_{1}(z+h)}}{\left(\gamma_{1}{ }^{2}-\gamma_{0}{ }^{2}\right) \rho^{3}}\right. \\
& \left.-\frac{e^{-\gamma_{1} R_{1}}}{\rho}\left[1+\frac{2}{\left(\gamma_{1}{ }^{2}-\gamma_{0}{ }^{2}\right) \rho^{2}}\left(1+\gamma_{1} \rho\right)\right]\right\}
\end{aligned}
$$

Here, we see that $\Pi_{x}$ has only three components: 1) a directwave component, 2) a modified mirror-image component, and 3) a lateral-wave component. Unlike Wu and King's results [3], [4], there is no false component that depends on the unphysical distance $z+h+\rho$.

At first glance, this procedure appears to be considerably more complicated than the procedure employed by $\mathrm{Wu}$ and King. Luckily, however, the general quasi-static range field components have already been derived for the four elementary dipoles (Wait [5], Weaver [6], Bannister [7], Bannister and Hart [8], Wait and Campbell [9], [10], and Sinha and Bhattacharya [11]) as have the lateral-wave expressions for the quasi-near, near-field, and far-field ranges $[1$, tables 3.2 ,

(5) 3.7, and 3.16]. Although displacement currents were ignored in most of these analyses, they can be included simply by replacing $\sigma_{1}$ by $\sigma_{1}+i \omega \epsilon_{1}$ (as long as $\left|n^{2}\right| \gg 1$ ). For the sake of convenience, the direct and modified mirror-image contribution to each dipole field-strength component is listed in Bannister [12, appendix]. For some components, these expressions are of very simple form $\left(H_{\phi} V E\right)$, while for other components, these expressions are quite complicated $\left(\mathrm{H}_{z}{ }^{V M}\right)$.

Thus the hardest part of the problem has already been solved. We can now use these previously derived results to obtain adequate formulas for the fields produced by submerged dipole sources subject to the conditions $\left|n^{2}\right| \gg 1$ and $\rho^{2} \gg(z+h)^{2}$.

The derivation procedure that we will follow is 1 ) to take the previously derived direct and modified mirror-image results and let $\rho$ be $\geqslant 3(z+h)$, remembering not to replace $R_{0}$ and $R_{1}$ by $\rho$ in the exponents, and 2 ) for the lateral-wave expressions, to let

$$
f(\rho, z+h)=f\left(\rho, 0^{+}\right) e^{-\gamma_{1}(z+h)}
$$

where $0^{+}$refers to an infinitesimal distance below the surface of the earth or water.

The quasi-static range $\left(\left|\gamma_{0} \rho\right| \ll 1\right)$ lateral-wave functions $f\left(\rho, 0^{+}\right)$can be obtained from Bannister [7] or [1, table 3.8] (with $\sigma_{1}$ replaced by $\sigma_{1}+i \omega \epsilon_{1}$ ). For the sake of convenience, the lateral-wave functions $f\left(\rho, 0^{+}\right) \exp \left[-\gamma_{1}(z+\right.$ $h)$ ] are presented in Table I. Note that, for some of the field-strength components, these expressions are identical to the quasi-near range $\left(\left|\gamma_{0} \rho\right| \ll 1 \ll\left|\gamma_{1} \rho\right|\right)$ results as given in $[1$, table 3.16]. Also, note that some of the signs are different than those of Bannister [7] and Kraichman [1]. This is because in this article we have inverted the coordinate system so that $z$ and $h$ are positive depths. 
TABLE I

$f\left(\rho, 0^{+}\right) e^{-r_{1}(z+h)}$ LATERAL-WAVE FORMULAS WHEN $\left|\gamma_{0} \rho\right| \ll 1\left[\left|n^{2}\right| \geq 10\right.$, $\left.\rho^{2}>(z+h)^{2}\right]$

\begin{tabular}{|c|c|c|c|c|c|c|}
\hline $\begin{array}{c}\text { Dipoie } \\
\text { Type }\end{array}$ & $E_{\rho}$ & $E_{\phi}$ & $E_{z}$ & $H_{\rho}$ & $\mathrm{H}_{\phi}$ & $\mathrm{H}_{2}$ \\
\hline VED & 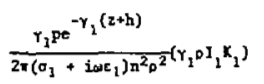 & 0 & $-\frac{p e^{-\gamma_{1}(2+h)}}{2 \pi\left(\sigma_{1}+i \omega \varepsilon_{1}\right) n^{2} \rho^{3}}$ & 0 & $\frac{\mathrm{pe}^{-Y_{2}(2+h)}}{2 \mathrm{~m}^{2} \mathrm{o}^{2}}$ & 0 \\
\hline VMD & 0 & $-\frac{3 \mathrm{i} \omega \mu_{0}{ }_{0}}{2 \tau\left(\gamma_{1}^{2}-r_{0}^{2}\right) \rho^{4}} e^{-r_{1}(z+h)}$ & 0 & $\frac{\mathrm{Ex}^{-Y_{1}(2+h)}}{2 \pi r_{1} p^{4}}\left(\frac{r_{1} \mathrm{pT}}{2}\right)$ & 0 & $\frac{9_{9 x}^{-\gamma_{1}}(z+h)}{2 \pi\left(r_{1}^{2}-\gamma_{0}^{2}\right) a^{5}}$ \\
\hline HED & $\frac{p \cos \phi e^{-r_{1}(z+h)}}{2 r\left(\sigma_{1}+i \omega \varepsilon_{1}\right) p^{3}}$ & $\frac{p \sin g e^{-\gamma_{1}(2+h)}}{\gamma\left(0_{1}+i \omega c_{1}\right) p^{3}}$ & $\frac{r_{1} p \cos p e^{-r_{1}(z+h)}}{2 r\left(\sigma_{1}+i \in \varepsilon_{1}\right) n^{2} p^{2}}\left(r_{1} p I_{1} x_{1}\right)$ & $-\frac{p \sin t e e^{-\gamma_{1}(z+h)}}{2 \pi Y_{1} p^{3}}\left(r_{1} \mathrm{pN}\right)$ & $\frac{2 \cdot \cos e^{-Y_{1}(2+h)}}{2 \pi r_{1} \rho^{3}}\left(r_{1} \rho I_{1} K_{1}\right)$ & $\frac{30 \sin \phi e^{-\gamma_{1}(z+h)}}{2 \pi\left(\gamma_{1}^{2}-\gamma_{0}^{2}\right) p^{4}}$ \\
\hline เม10 & $\begin{array}{l}-\frac{i \omega \nu_{0} \Phi \cos \phi}{2 \pi \mu^{\rho^{3}}}-\left(\gamma_{1} p I_{1} K_{1}\right) \\
x e^{-\gamma_{1}(z \cdot h)}\end{array}$ & $\begin{array}{l}-\frac{i \omega \nu_{0} m \sin \phi}{2 \pi Y_{1} p^{3}}\left(Y_{1} p W\right) \\
\times e^{-Y_{1}(t+h)}\end{array}$ & $\frac{\operatorname{lis}_{0}=\cos \theta}{2 \pi^{2} \rho^{2}} e^{-\gamma_{1}(2+h)}$ & $\frac{n \sin \phi}{x_{0}^{3}}\left(1-\frac{6}{r_{1}^{2} \rho^{2}}\right) e^{-r_{1}(z+h)}$ & $-\frac{m \cos \phi}{2 \pi \rho^{3}}\left(1-\frac{3}{\gamma_{1}^{2} \rho^{2}}\right) e^{-r_{2}(z+h)}$ & $-\frac{a \sin \phi e^{-r_{1}(z+h)}}{2 \pi \gamma_{1} e^{4}}\left(\frac{r_{1} \rho T}{2}\right)$ \\
\hline
\end{tabular}

*Argument of modified Bessel fumctions is $r_{1} \mathrm{p} / 2$.

Approximately half of the field-component formulas as listed in Table I involve products of modified Bessel functions of argument $\gamma_{1} \rho / 2$. The functions $T$ and $W$ are defined by

$$
\begin{aligned}
T= & \left\{16 I_{1} K_{1}+\gamma_{1}{ }^{2} \rho^{2}\left[I_{1} K_{1}-I_{0} K_{0}\right]\right. \\
& \left.+4 \gamma_{1} \rho_{1}\left[I_{1} K_{0}-I_{0} K_{1}\right]\right\}
\end{aligned}
$$

and

$$
W=\left[3 I_{1} K_{1}-\frac{\gamma_{1} \rho}{2}\left(I_{0} K_{1}-I_{1} K_{0}\right)\right] .
$$

Numerical values for these functions have been provided by Bannister [7]. When $\left|\gamma_{1} \rho\right| \geqslant 4$, the function $\gamma_{1} \rho I_{1} K_{1} \approx 1$, while when $\left|\gamma_{1} \rho\right| \geqslant 6$, the function $\gamma_{1} \rho W \approx 2$. Furthermore, when $\left|\gamma_{1} \rho\right| \geqslant 10$, the function $\gamma_{1} \rho T / 2 \approx 3$.

As an example of our derivation procedure, consider the HED $E_{o}$ component. The lateral-wave $E_{o}$ component for the quasi-static range can be obtained from Table I or [5, eq. (67)]. It is equal to

$$
E_{\rho}{ }^{H E} \approx \frac{p \cos \phi}{2 \pi\left(\sigma_{1}+i \omega \epsilon_{1}\right) \rho^{3}} e^{-\gamma_{1}(z+h)} .
$$

The lateral-wave $E_{\rho}$ component for the nearfield range can be obtained from [1, table 3.7] (with $\sigma_{1}$ replaced by $\left.\sigma_{1}+i \omega \epsilon_{1}\right)$, or from [5, eqs. (23) and (32)]. Therefore

$$
E_{\rho}^{H E} \approx \frac{p \cos \phi e^{-\gamma_{1}(z+h)} e^{-\gamma_{0} \rho}}{2 \pi\left(\sigma_{1}+i \omega \epsilon_{1}\right) \rho^{3}}\left(1+\gamma_{0} \rho+\gamma_{0}{ }^{2} \rho^{2}\right)
$$

The lateral-wave $E_{\rho}$ component for the farfield range can be obtained from [1, table 3.2] (with $\sigma_{1}$ replaced by $\sigma_{1}+i \omega \epsilon_{1}$ ), or from [5, eqs. (13) and (32). Thus

$$
E_{\rho}^{H E} \approx \frac{p \cos \phi e^{-\gamma_{1}(z+h)} e^{-\gamma_{0} \rho}}{2 \pi\left(\sigma_{1}+i \omega \epsilon_{1}\right) \rho^{3}}\left(\gamma_{0}^{2} \rho^{2} F\right)
$$

where

$$
F=F\left(w_{0}\right)=1-i\left(\pi w_{0}\right)^{1 / 2} e^{-w_{0}} \operatorname{erfc}\left(i w_{0}^{1 / 2}\right)
$$

is the Sommerfeld surface-wave attenuation function and

$$
w_{0}=-\frac{\gamma_{0} \rho}{2 n^{2}}
$$

is the Sommerfeld numerical distance. For small numerical distances $F\left(w_{0}\right) \approx 1$, while for larger numerical distances $F\left(w_{0}\right) \approx-1 /\left(2 w_{0}\right)$. Various approximate expressions for $F\left(w_{0}\right)$ are provided by Norton [13] , [14] and Li [15].

When $\left|n^{2}\right| \gg 1$, the range of validity (14) and (15) overlap when $\left|\gamma_{0} \rho\right| \ll 1$. Similarly, when $\left|w_{0}\right| \ll 1$ and $\left|\gamma_{0} \rho\right| \gg 1$, the range of validity of (15) and (16) overlap. Therefore, we can simply combine (14), (15), and (16) to obtain an expression for the $E_{\rho}$ lateral-wave component valid from the quasi-static to the farfield range. Therefore, for $\left|n^{2}\right| \gg 1$ and $\rho^{2} \gg(z+h)^{2}$

$$
E_{\rho}^{H E} \approx \frac{p \cos \phi e^{-\gamma_{1}(z+h)} e^{-\gamma_{0} \rho}}{2 \pi\left(\sigma_{1}+i \omega \epsilon_{1}\right) \rho^{3}}\left(1+\gamma_{0} \rho+\gamma_{0}{ }^{2} \rho^{2} F\right) .
$$

The direct and modified mirror-image contribution to each dipole field-strength component is listed in Bannister [12, appendix]. These expressions are valid for $\left|n^{2}\right| \gg 1$. When 
$\rho \geqslant 3(z+h)$, the HED $E_{\rho}$ expression reduces to

$$
\begin{gathered}
E_{\rho} H E \approx \frac{p \cos \phi}{2 \pi\left(\sigma_{1}+i \omega \epsilon_{1}\right) \rho^{3}}\left\{\left(1+\gamma_{1} \rho\right) e^{-\gamma_{1} R_{0}}\right. \\
-\left(3+3 \gamma_{1} \rho^{2}+\gamma_{1}{ }^{2} \rho^{3}\right)\left[\frac{(z-h)^{2}}{2 \rho^{2}} e^{-\gamma_{1} R_{0}}\right. \\
\left.\left.+\frac{(z+h)^{2}}{2 \rho^{2}} e^{-\gamma_{1} R_{1}}\right]\right\} .
\end{gathered}
$$

We can now combine (19) and (20) and obtain an expression for the HED $E_{\rho}$ component valid at almost any range from the source subject to the conditions that $\left|n^{2}\right| \gg 1$ and $\rho \geqslant 3(z+h)$. The final expression is

$$
\begin{aligned}
E_{\rho}^{H E} \approx & \frac{p \cos \phi}{2 \pi\left(\sigma_{1}+i \omega \epsilon_{1}\right) \rho^{3}}\left\{\left(1+\gamma_{0} \rho\right.\right. \\
& \left.+\gamma_{0}^{2} \rho^{2} F\right) e^{-\gamma_{0} \rho} e^{-\gamma_{1}(z+h)}+\left(1+\gamma_{1} \rho\right) e^{-\gamma_{1} R_{0}} \\
& -\left(3+3 \gamma_{1} \rho+\gamma_{1}^{2} \rho^{2}\right)\left[\frac{(z-h)^{2}}{2 \rho^{2}} e^{-\gamma_{1} R_{0}}\right. \\
& \left.\left.+\frac{(z+h)^{2}}{2 \rho^{2}} e^{-\gamma_{1} \cdot R_{1}}\right]\right\}
\end{aligned}
$$

When $\rho^{2} \gg(z+h)^{2}$, the last two terms of (21) are negligible compared with the first two, resulting in

$$
\begin{aligned}
E_{\rho}{ }^{H E} \approx & \frac{p \cos \phi}{2 \pi\left(\sigma_{1}+i \omega \epsilon_{1}\right) \rho^{3}}\left[\left(1+\gamma_{0} \rho\right.\right. \\
& \left.\left.+\gamma_{0}{ }^{2} \rho^{2} F\right) e^{-\gamma_{0} \rho} e^{-\gamma_{1}(z+h)}+\left(1+\gamma_{1} \rho\right) e^{-\gamma_{1} R_{0}}\right]
\end{aligned}
$$

which is identical to Wu and King's result [2] for $\left|n^{2}\right| \gg 1$ and $\rho^{2} \gg(z+h)^{2}$. Wu and King have shown that this simple formula is in excellent agreement with the exact results obtained from the numerical integration of the Sommerfeld integrals.

New formulas for the electric and magnetic fields produced by the four elementary dipole antennas are presented in Tables II and III for the subsurface-to-subsurface propagation case. All of these formulas have been obtained from previous derived results according to the procedure outlined above and are strictly valid for $\left|n^{2}\right| \gg 1$ and $\rho^{2} \gg(z+h)^{2}$. (However, for most cases, the requirements that $\left|n^{2}\right| \geqslant 10$ and $\rho \geqslant$ $3(z+h)$ are sufficient.)

It should be noted that for many (but not all) cases, the range of validity of the formulas presented in Tables II and III can be extended down to $\rho \approx(z+h)$ if the direct and modified mirror-image terms in these equations are replaced by the equations listed in the appendix of Bannister [12] For example, the HED $E_{\rho}$ expression (21) would be replaced by

$$
\begin{aligned}
E_{\rho}^{H E} \approx & \frac{p \cos \phi}{4 \pi\left(\sigma_{1}+i \omega \epsilon_{1}\right)}\left\{\frac { 2 } { \rho ^ { 3 } } \left(1+\gamma_{0} \rho\right.\right. \\
& \left.+\gamma_{0}{ }^{2} \rho^{2} F\right) e^{-\gamma_{0} \rho} e^{-\gamma_{1}(z+h)}+\left[\left(3 \cos ^{2} \psi_{0}\right.\right. \\
& \left.-1)\left(1+\gamma_{1} R_{0}\right)-\gamma_{1}{ }^{2} R_{0}{ }^{2} \sin ^{2} \psi_{0}\right] \frac{e^{-\gamma_{1} R_{0}}}{R_{0}{ }^{3}} \\
& \left.-\left(3+3 \gamma_{1} R_{1}+\gamma_{1}{ }^{2} R_{1}{ }^{2}\right) \sin ^{2} \psi_{1} \frac{e^{-\gamma_{1} R_{1}}}{R_{1}{ }^{3}}\right\}
\end{aligned}
$$

where $\sin \psi_{0}=(z-h) / R_{0}, \cos \psi_{0}=\rho / R_{0}$ and $\sin \psi_{1}=$ $(z+h) / R_{1}$.

It should also be noted that the two media can be inverted and the air (medium 0 ) replaced by the earth's crust (of conductivity $\sigma_{2}$ and dielectric constant $\epsilon_{2}$ ). The same equations (Tables I-III) can be utilized, as long as $\left|n_{2}{ }^{2}\right|=\left|\gamma_{1}{ }^{2} / \gamma_{2}{ }^{2}\right| \geqslant$ 10 and $\rho \geqslant 3(z+h)$, simply by replacing $i \omega \epsilon_{0}$ by $\sigma_{2}+i \omega \epsilon_{2}$.

In the ELF band, for the air/sea case, the measurement distance will be much less than a free-space wavelength (i.e., $\left.\left|\gamma_{0} \rho\right| \ll 1\right)$. Therefore, the lateral-wave portion of the equations presented in Tables II and III can be replaced by those listed in Table I, resulting in even further simplification (e.g., the HED $E_{\rho}$ lateral-wave formula (19) can be replaced by (14)). However, for the ELF sea/seabed case, the wavelength in the sea bed will usually be comparable to the measurement distance, and the general formulas presented in Tables II and III are applicable.

\section{RANGE OF VALIDITY OF LATERAL-WAVE FORMULAS}

As we have previous mentioned, the attenuation factor $\exp \left[-\gamma_{1}(z+h)\right]$ for the lateral wave in the quasi-static range emerges only from the integral $N$, as given in (7).

When $\left|\gamma_{1} \rho\right| \gg 1$ and $\rho \gg(z+h)$, Wait and Campbell [10] have shown that the modified Bessel functions may be replaced by only the first terms in their respective asymptotic expansions to obtain adequate HMD expressions for the quasi-near range. On the other hand, Sinha and Bhattacharya [11] have shown, for the VMD case, the first two terms of the modified Bessel function's asymptotic expansion must be employed. This indicates that the range of validity of the lateral-wave expressions for the quasi-static range will not be the same for all field components.

As an example, consider the HED $E_{\rho}$ lateral-wave component for the quasi-static range, which can be expressed as

$$
E_{\rho}^{L W} \approx-\frac{p \cos \phi}{2 \pi\left(\sigma_{1}+i \omega \epsilon_{1}\right) \rho} \frac{\partial}{\partial \rho} \int_{0}^{\infty} e^{-u_{1}(z+h)} J_{0}(\lambda \rho) d \lambda .
$$

When $\left|\gamma_{1} \rho\right| \gg 1$ and $\rho \gg(z+h)$, the usual procedure is to replace $u_{1}$ in the exact integral expressions by $\gamma_{1}$, the propa- 


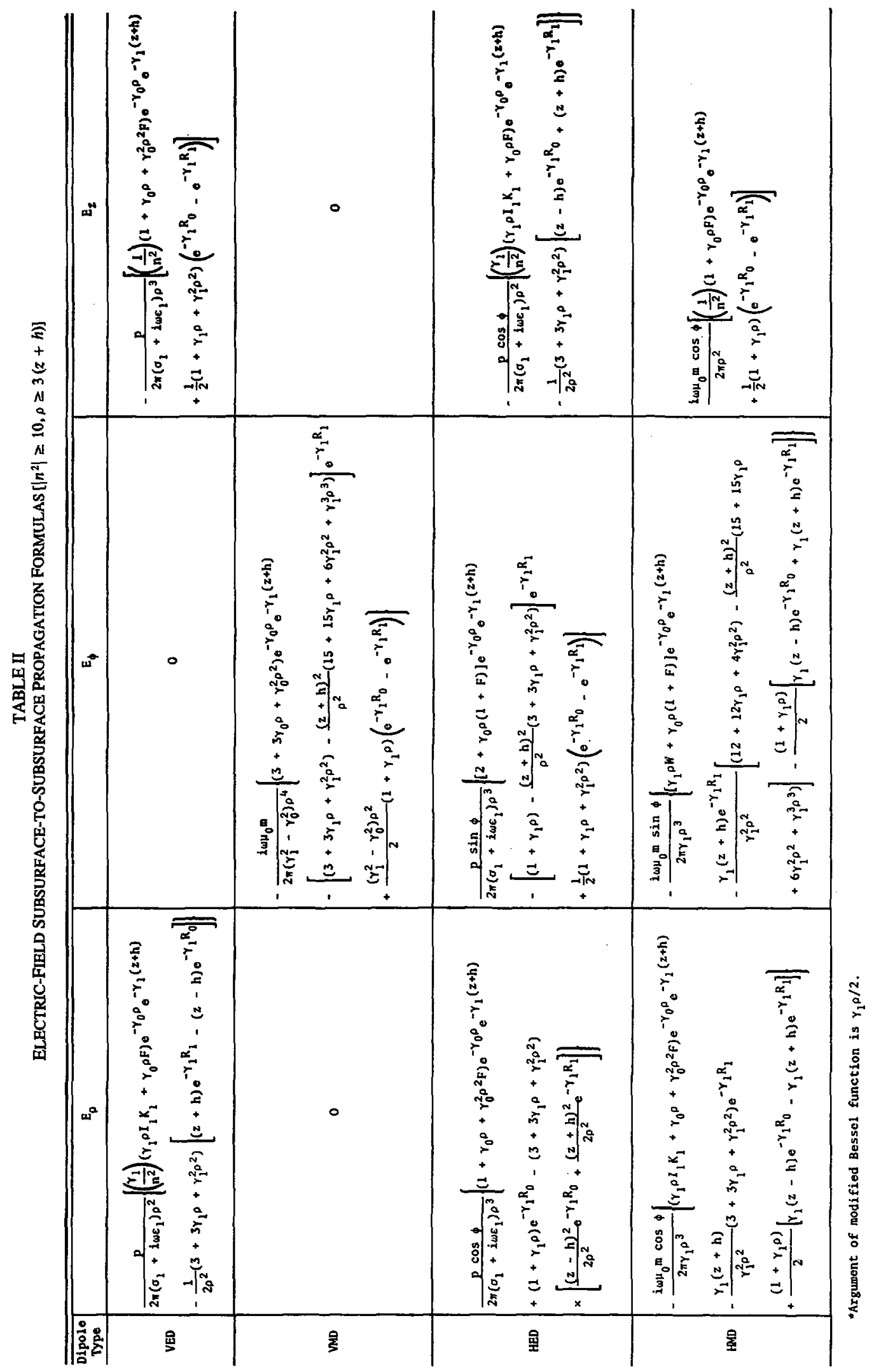




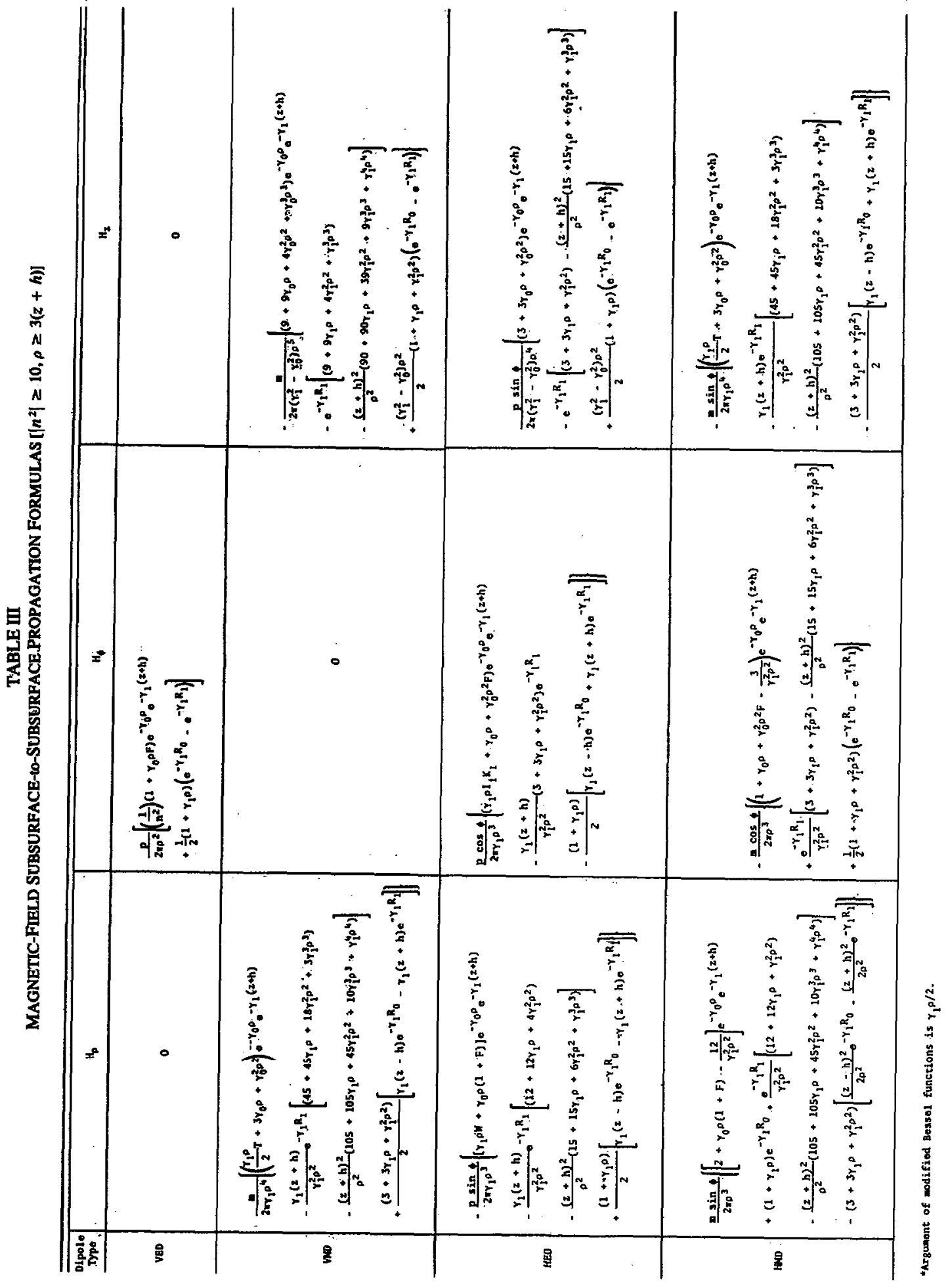


gation constant in the earth, resulting in

$$
E_{\rho}{ }^{L W} \approx-\frac{p \cos \phi e^{-\gamma_{1}(z+h)}}{2 \pi\left(\sigma_{1}+i \omega \epsilon_{1}\right) \rho} \frac{\partial}{\partial \rho} \int_{0}^{\infty} J_{0}(\lambda \rho) d \lambda
$$

Since this integral is equal to $1 / \rho$ (Erdélyi [16])

$$
E_{\rho}^{L W} \approx \frac{p \cos \phi e^{-\gamma_{1}(z+h)}}{2 \pi\left(\sigma_{1}+i \omega \epsilon_{1}\right) \rho^{3}}=f\left(\rho, 0^{+}\right) e^{-\gamma_{1}(z+h)} .
$$

So far, we do not know exactly what values $\left|\gamma_{1} \rho\right|$ and $\rho /(z+h)$ must have in order to utilize (26) or any of the other quasi-static range lateral-wave formulas presented in Table 1 .

As a first-order approximation, we will let

$$
u_{1}=\left(\lambda^{2}+\gamma_{1}^{2}\right)^{1 / 2} \approx \gamma_{1}+\frac{\lambda^{2}}{2 \gamma_{1}}
$$

so that

$$
\begin{aligned}
e^{-u_{1}(z+h)} & \approx e^{-\gamma_{1}(z+h)} e^{-\lambda^{2}(z+h) / 2 \gamma_{1}} \\
& \approx e^{-\gamma_{1}(z+h)}\left[1-\frac{\lambda^{2}(z+h)}{2 \gamma_{1}}\right] .
\end{aligned}
$$

Inserting (28) into (24) results in

$$
\begin{aligned}
E_{\rho}{ }^{L W} \approx & -\frac{p \cos \phi e^{-\gamma_{1}(z+h)}}{2 \pi\left(\sigma_{1}+i \omega \epsilon_{1}\right) \rho} \frac{\partial}{\partial \rho}\left[\int_{0}^{\infty} J_{0}(\lambda \rho) d \lambda\right. \\
& \left.-\frac{(z+h)}{2 \gamma_{1}} \int_{0}^{\infty} \lambda^{2} J_{0}(\lambda \rho) d \lambda\right] .
\end{aligned}
$$

Since the second integral is equal to $-1 / \rho^{3}$ (Erdélyi [16])

$$
E_{\rho}{ }^{L W} \approx \frac{p \cos \phi e^{-\gamma_{1}(z+h)}}{2 \pi\left(\sigma_{1}+i \omega \epsilon_{1}\right) \rho^{3}}\left[1+\frac{3(z+h)}{2 \gamma_{1} \rho^{2}}\right] .
$$

It can easily be shown that the error incurred in neglecting the second term is less than $1 \mathrm{~dB}$ if the quantity

$$
\left|\left(\gamma_{1} \rho\right)\left(-\frac{\rho}{z+h}\right)\right| \geqslant 4 c_{1}
$$

where $c_{1}=3$ for HED $E_{\rho}$ component. That is, to a firstorder approximation, it is the product of $\left|\gamma_{1} \rho\right| \times \rho /(z+$ $h$ ) that must exceed a specified number to accurately utilize (26) or any of the other quasi-static range lateral-wave formulas presented in Table I. For example, if $\rho=5(z+h)$, we require $\left|\gamma_{1} \rho\right| \geqslant 2.4$ to use (26).

The most severe restriction will be for the VMD $H_{z}$ component. Following the same procedure as in the derivation of the HED $E_{\rho}$ component, we can express the VMD $H_{z}$ compo- nent as

$$
H_{z}{ }^{V M} \approx-\frac{9 m e^{-\gamma_{1}(z+h)}}{2 \pi\left(\gamma_{1}{ }^{2}-\gamma_{0}{ }^{2}\right) \rho^{5}}\left[1+\frac{25(z+h)}{2 \gamma_{1} \rho^{2}}\right] .
$$

Again, it can easily be shown that the error incurred in neglecting the second term is less than $1 \mathrm{~dB}$ if (31) is satisfied, with $c_{1}=25$ for the VMD $H_{z}$ component. For example, if $\rho=$ $5(z+h)$, then we require $\left|\gamma_{1} \rho\right| \geqslant 20$ to employ the VMD $H_{z}$ lateral-wave formula for the quasi-static range presented in Table I.

The values of $c_{1}$ and range where $f(\rho, z+h)$ can be replaced by $f\left(\rho, 0^{+}\right) \exp \left[-\gamma_{1}(z+h)\right]$, i.e., the range where the quasistatic range lateral-wave formulas presented in Table I can be used) are presented in Table IV for each field-strength compnent. Here, we see that for the HED and HMD $E_{\rho}, E_{z}$, and $H_{\phi}$ components and the VED $E_{\rho}$ and $H_{\phi}$ components $\left(c_{1}=\right.$ $3)$, the condition $\left|\gamma_{1} \rho^{2} /(z+h)\right| \geqslant 12$ must be satisfied, while for the HED $H_{z}$ and VMD $E_{\phi}$ and $H_{\rho}$ components $\left(c_{1}=15\right)$, the condition $\left|\gamma_{1} \rho^{2} /(z+h)\right| \geqslant 60$ must be satisfied.

This restriction (31) also applies to Wu and King's results [2]-[4] and to the quasi-near and nearfield range subsurfaceto-subsurface propagation equations tabulated in Kraichman [1].

To a first-order approximation, the range of validity of the equations listed in Table I can be extended by multiplying the field-component expression by the quantity

$$
1+\frac{c_{1}(z+h)}{2 \gamma_{1} \rho^{2}}
$$

where the value of $c_{1}$ for each component is given in Table IV. For example, the HED $H_{z}$ component listed in Table $I$ is

$$
H_{z}^{H E} \approx \frac{3 p \sin \phi e^{-\gamma_{1}(z+h)}}{2 \pi\left(\gamma_{1}{ }^{2}-\gamma_{0}{ }^{2}\right) \rho^{4}} .
$$

Since $c_{1}=15$ for this component (Table IV), then the extended form is

$$
H_{z}^{H E} \approx \frac{3 p \sin \phi e^{-\gamma_{1}(z+h)}}{2 \pi\left(\gamma_{1}{ }^{2}-\gamma_{0}^{2}\right) \rho^{4}}\left[1+\frac{15(z+h)}{2 \gamma_{1} \rho^{2}}\right] .
$$

\section{ANALYTICAL CONFIRMATION OF VMD $H_{z}$ NULL FOUND BY FRASER-SMITH AND BUBENIK}

A few years ago, Fraser-Smith and Bubenik [17] numerically evaluated the exact Sommerfeld integrals and found a rather deep null in the VMD $H_{z}$ component for the subsurface-tosurface propagation case [17, fig. 6]. For their particular situation, the frequency was $100 \mathrm{~Hz}$, the VMD source depth of burial was $100 \mathrm{~m}$, and the null occurred at a distance $\rho \approx 250 \mathrm{~m}$. Since the skin depth $\delta$ in seawater at $100 \mathrm{~Hz}$ is $\approx 25 \mathrm{~m}, \rho / \delta \approx 10$ and $h / \delta \approx 4$. To our knowledge, this null has not been analytically confirmed. The subsurfaceto-surface VMD $H_{z}$ component is equal to (from Table III, 
TABLE IV

RANGE OF VALIDITY FOR QUASI-STATIC RANGE LATERAL-WAVE FORMULAS OF TABLE I

(where $\left.\left.f(\rho, z+h)=f(\rho, 0)^{+}\right) e^{-\gamma_{1}(z+h)}\right)$

\begin{tabular}{c|c|c|c|c}
\hline \multicolumn{2}{c|}{ Field-Strength Components } & \multirow{2}{*}{$c_{1}$} & $\left|\left(\gamma_{1} \rho\right)\left(\frac{\rho}{\mathrm{z}+\mathrm{h}}\right)\right|$ \\
\hline HED and HMD & VED & WMD & ${ }^{\prime}$ \\
\hline$E_{\rho}, \mathrm{E}_{Z}, \mathrm{H}_{\phi}$ & $\mathrm{E}_{\rho}, \mathrm{H}_{\phi}$ & -- & 3 & $\geq 12$ \\
$\mathrm{E}_{\phi}, \mathrm{H}_{\rho}$ & -- & -- & 6 & $\geq 24$ \\
-- & $\mathrm{E}_{Z}$ & -- & 9 & $\geq 36$ \\
$\mathrm{H}_{Z}$ & -- & $\mathrm{E}_{\phi}, \mathrm{H}_{\rho}$ & 15 & $\geq 60$ \\
-- & -- & $\mathrm{H}_{Z}$ & 25 & $\geq 100$ \\
\hline
\end{tabular}

with $z=0$ )

$$
\begin{aligned}
H_{z} V M \approx & -\frac{m}{2 \pi\left(\gamma_{1}{ }^{2}-\gamma_{0}{ }^{2}\right) \rho^{5}}\left\{\left(9+9 \gamma_{0} \rho+4 \gamma_{0}{ }^{2} \rho^{2}\right.\right. \\
& \left.+\gamma_{0}{ }^{3} \rho^{3}\right) e^{-\gamma_{0} \rho} e^{-\gamma_{1} h}-e^{-\gamma_{1} D}\left[\left(9+9 \gamma_{1} \rho\right.\right. \\
& \left.+4 \gamma_{1}{ }^{2} \rho^{2}+\gamma_{1}{ }^{3} \rho^{3}\right)-\frac{h^{2}}{\rho^{2}}\left(90+90 \gamma_{1} \rho\right. \\
& \left.\left.\left.+39 \gamma_{1}{ }^{2} \rho^{2}+9 \gamma_{1}{ }^{3} \rho^{3}+\gamma_{1}{ }^{4} \rho^{4}\right)\right]\right\}
\end{aligned}
$$

where $D^{2}=\rho^{2}+h^{2}$.

For $f=100 \mathrm{~Hz}$ and $\rho=250 \mathrm{~m},\left|\gamma_{0} \rho\right| \ll 1$ and the $\gamma_{0} \rho$ terms in the lateral-wave portion of (36) are negligible. Furthermore, since the quantity $\left|\gamma_{1} \rho^{2} / h\right| \approx 35$, which is $<100$ (see Table IV), (33) must be employed. The resulting expression for $H_{z}$ is

$$
H_{z}{ }^{V M} \approx-\frac{9 m e^{-\gamma_{1} h}}{2 \pi \gamma_{1}{ }^{2} \rho^{5}} H_{z}{ }^{\prime}
$$

where

$$
\begin{aligned}
H_{z}{ }^{\prime} \approx & \left(1+\frac{25 h}{2 \gamma_{1} \rho^{2}}\right)-\left\{\frac { e ^ { - \gamma _ { 1 } ( D - h ) } } { 9 } \left[\left(9+9 \gamma_{1} \rho\right.\right.\right. \\
& \left.+4 \gamma_{1}{ }^{2} \rho^{2}+\gamma_{1}{ }^{3} \rho^{3}\right)-\frac{h^{2}}{\rho^{2}}\left(90+90 \gamma_{1} \rho\right. \\
& \left.\left.\left.+39 \gamma_{1}{ }^{2} \rho^{2}+9 \gamma_{1}{ }^{3} \rho^{3}+\gamma_{1}{ }^{4} \rho^{4}\right)\right]\right\} .
\end{aligned}
$$

For $\rho / \delta \approx 10$, the dominant terms will be the $\gamma_{1}{ }^{3} \rho^{3}$ and $\gamma_{1}{ }^{4} \rho^{4}$ terms. To a first-order approximation, the $\gamma_{1}{ }^{3} \rho^{3}$ terms will cancel and since $\gamma_{1}{ }^{4} \rho^{4}=-4(\rho / \delta)^{4}$

$$
\begin{aligned}
H_{z}{ }^{\prime} \approx & 1+\frac{25 h}{2 \gamma_{1} \rho^{2}} \\
& -\frac{4}{9}\left(\frac{h}{\rho}\right)^{2}\left(\frac{\rho}{\delta}\right)^{4} e^{-(D-h) / \delta} e^{-i(D-h) / \delta} .
\end{aligned}
$$

This equation will be at a minimum near $(D-h) / \delta=2 \pi$, which corresponds to $\rho \approx 240 \mathrm{~m}$. The normalized VMD vertical magnetic field $\left(H_{z}\right.$ from (38)) is plotted in Fig. 1 versus the horizontal distance $\rho$. For this figure, we can see that a rather deep null $(\approx 20$-dB drop in field strength compared to the asymptotic value) occurs at a range of $\approx 240$ $\mathrm{m}$. This null is clearly due to the destructive inteference between the direct and lateral waves.

\section{CONCLUSIONS}

New formulas for the electric and magnetic fields produced by the four elementary dipole antennas have been developed for the subsurface-to-subsurface propagation case. These formulas have been obtained completely from previously derived results. The main restrictions in their use are 1) the square of the index of refraction is greater than or equal to 10 and 2) the horizontal separation is greater than or equal to 3 times the sum of the depth of burial of the transmitting and receiving point sources. An additional restriction must also be applied to the lateral-wave components. The condition $\left|\gamma_{1} \rho^{2} /(z+h)\right| \geqslant 4 c_{1}$ must be satisfied, where $c_{1}=3,6,9$, 15 , or 25 , depending on the particular field-strength component. This, restriction also applied to Wu and King's recently derived results [2]-[4] and to the subsurface-to-subsurface propagation equations tabulated in Kraichman [1].

These new formulas are also an improvement over Wu and King's results [3] , [4]. They contain no false component that depends on the unphysical distance $z+h+\rho$. Wu and King's use of this unphysical distance leads to errors in the range where the various waves interfere, either constructively or destructively, with each other.

An analytical confirmation of the Fraser-Smith and Bubenik VMD $H_{z}$ null had also been accomplished. This null is clearly due to the destructive interference between the direct and lateral waves.

The range of validity of the equations tabulated in this article can extended down to $\rho \approx(z+h)$ for many cases if the direct and modified mirror-image terms in these equations are replaced with the equations listed in Bannister [12, appendix].

It should be noted that the two media can be inverted and the air replaced by the earth's crust (of conductivity $\sigma_{2}$ and dielectric constant $\epsilon_{2}$ ). The same equations (Table IIV) can be utilized, as long as $\left|n_{2}{ }^{2}\right|=\mid \gamma_{1}{ }^{2} / \gamma_{2}{ }^{2} i \geqslant 10$ and $\rho \geqslant 3(z+h)$, simply by replacing $i \omega \epsilon_{0}$ by $\sigma_{2}+i \omega \epsilon_{2}$.

Although the field component expressions presented in this article are valid at any frequency, they are especially useful at ELF for the air/sea and sea/seabed propagation cases.

In addition to the results presented in this article, we have also developed new formulas [18] that extend Norton's farfield equations to the quasi-nearfield range. Air-to-air, subsurface-to-air, air-to-subsurface, and surface-to-surface propagation were investigated. We have also employed image theory to derive expressions valid at any range from the source for air-to-air propagation [19] .

\section{APPENDIX}

\section{COMPARISON OF BANNISTER AND WU AND KING RESULTS}

In their first article [2], Wu and King developed a new simple and very accurate formula for the radial field $\left(E_{\rho}\right)$ 


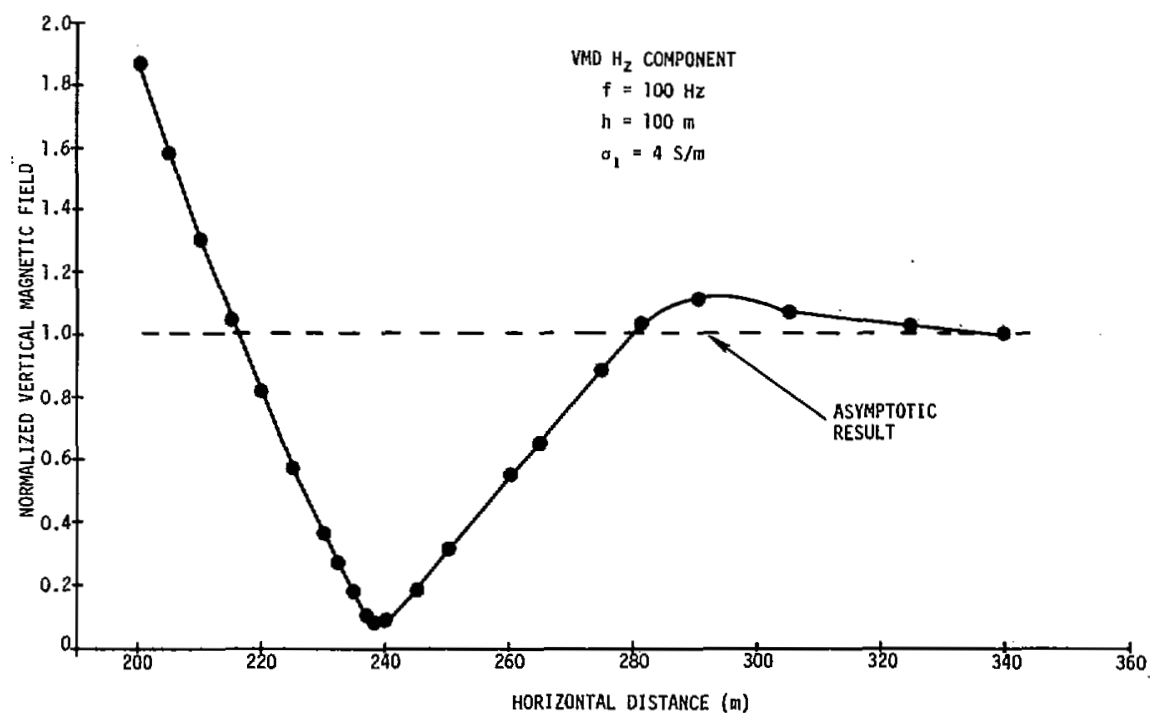

Fig. 1. Normalized VMD vertical magrietic field versus distance for $f=$ $100 \mathrm{~Hz}, h=100 \mathrm{~m}$, and $\sigma_{1}=4 \mathrm{~S} / \mathrm{m}$.

of a HED in a dissipative or dielectric half-space near its boundary with air. They examined in detail the inteference patterns generated by the direct and lateral waves that originate at the dipole for three values of $\epsilon_{1}$, numerous values of $\sigma_{1}$, and a wide range of frequencies. They confirmed the accuracy of the new formula by comparison with numerically evaluated Sommerfeld integral results.

In their second article [3], Wu and King developed new simple formulas for the transverse $\left(E_{\phi}\right)$ and vertical $\left(E_{z}\right)$ electric-field components generated by a buried HED source. They then compared all three electric-field components with numerical integration results for the case where $\sigma_{1}=3.5$ $\mathrm{S} / \mathrm{m}, \epsilon_{1}=45 \epsilon_{0}$, and $f=600 \mathrm{MHz}$ (their Fig. 1.). The agreement between the simple-formula and numerical-integration results was excellent for the radial component when $\left|\gamma_{1} \rho\right|>$ 2.8 and $\rho>2(z+h)$. However, substantial agreement between the simple-formula and numerical-integration transverse and vertical components was not achieved until $\left|\gamma_{1} \rho\right|>11.5$ and $\rho>8(z+h)$. Furthermore, the simple formulas predicted a dip in the transverse components and no dip in the vertical component near $\left|\gamma_{1} \rho\right| \approx 8$, while the numerical-integration results predicted the opposite.

In attempting to explain the major discrepancy between the HED $E_{\phi}$ and $E_{z}$ simple-formula and numerical-integration results near $\left|\gamma_{1} \rho\right| \approx 8$ of [3, fig. 1], Wu and King stated that very small changes in frequency significantly alter the interference pattern so that close agreement in a small range near such a region cannot be expected. This statement contradicts the $E_{\rho}$ results presented in their first article (Wu and King [2]), where substantial agreement between the simpleformula and numerical-integration results was achieved in the interference region when $\left|n^{2}\right| \geqslant 1$ and $\rho \geqslant 5(z+h)$.

They also noted, at greater distances where the lateral wave dominates, the $E_{\rho}, E_{\phi}$, and $E_{z}$ expression were highly accurate. They certainly should be highly accurate at the greater distances because their lateral-wave formulas are es- sentially equivalent to Wait's results [5], which have been successfully utilized for over 20 years.

The major value of Wu and Kings' work is in determining adequate field-strength expressions in the range where the lateral and direct waves interfere (either constructively or destructively). They have succeeded with the $\operatorname{HED} E_{\rho}$ component. However, they have made some errors in their derivation of the other five HED components.

The HED $E_{\phi}$ and $E_{z}$ expressions derived in this article (Table II) are in very good agreemeit with the numericalintegration results presented in fig. 1 of $W u$ and King [3] when $\rho \geqslant 3(z+h)$. They correctly predict a substantial dip in the $E_{z}$ component (due to interference between the direct, mirror-image, and lateral waives) near $\left|\gamma_{1} \rho\right| \approx 8$ and no dip in the $E_{\phi}$ component at this range (which is directly opposite to Wu and Kings' simple-formula results).

To see where Wu and King erred, we will compare their $E_{\phi}$ and $E_{z}$ formulas with the expressions listed in Table II for the situation where the Sommerfeld numerical distance is small (i.e., $F \approx 1), \rho \geqslant 5(z+h)$, and $\left|\gamma_{1} \rho\right| \geqslant 4$. For this situation, the $E_{\phi}$ and $E_{z}$ exprêssions in Table II reduce to

$$
\begin{aligned}
E_{\phi} H E \approx & \frac{p \sin \phi}{2 \pi\left(\sigma_{1}+i \omega \epsilon_{1}\right) \rho^{3}}\left[2\left(1+\gamma_{0} \rho\right) e^{-\gamma_{0} \rho} e^{-\gamma_{1}(z+h)}\right. \\
& -\left(1+\gamma_{1} \rho\right) e^{-\gamma_{1} R_{1}}+\frac{1}{2}\left(1+\gamma_{1} \rho\right. \\
& \left.\left.+\gamma_{1}{ }^{2} \rho^{2}\right)\left(e^{-\gamma_{1} R_{0}}-e^{-\gamma_{1} R_{1}}\right)\right]
\end{aligned}
$$

and

$$
\begin{aligned}
E_{z} H E \approx & -\frac{p \cos \phi}{2 \pi\left(\sigma_{1}+i \omega \epsilon_{1}\right) \rho^{2}}\left\{\frac{\gamma_{1}}{n^{2}}(1\right. \\
& \left.+\gamma_{0} \rho\right) e^{-\gamma_{0} \rho} e^{-\gamma_{1}(z+h)}-\frac{1}{2 \rho^{2}}\left(3+3 \gamma_{1} \rho\right. \\
& \left.\left.+\gamma_{1}{ }^{2} \rho^{2}\right)\left[(z-h) e^{-\gamma_{1} R_{0}}+(z+h) e^{-\gamma_{1} R_{1}}\right]\right\} .
\end{aligned}
$$


When $F \approx 1, \rho \geqslant 5(z+h)$, and $\left|\gamma_{1} \rho\right| \geqslant 4, W u$ and King's $E_{\phi}$ and $E_{z}$ expressions [3] reduce to

$$
\begin{aligned}
E_{\phi} H E \approx & \frac{p \sin \phi}{2 \pi\left(\sigma_{1}+i \omega \epsilon_{1}\right) \rho^{3}}[2(1 \\
& \left.+\gamma_{0} \rho\right) e^{-\gamma_{0} \rho} e^{-\gamma_{1}(z+h)}-\left(2+2 \gamma_{1} \rho\right. \\
& \left.+\gamma_{1}{ }^{2} \rho^{2}\right) e^{-\gamma_{1}(z+h+\rho)}+\left(1+\gamma_{1} \rho\right. \\
& \left.+\gamma_{1}{ }^{2} \rho^{2}\right) e^{-\gamma_{1} R_{1}}+\frac{1}{2}\left(1+\gamma_{1} \rho\right. \\
& \left.\left.+\gamma_{1}{ }^{2} \rho^{2}\right)\left(e^{-\gamma_{1} R_{0}}-e^{-\gamma_{1} R_{1}}\right)\right]
\end{aligned}
$$

and

$$
\begin{aligned}
E_{z} H E \approx & \frac{p \cos \phi}{2 \pi\left(\sigma_{1}+i \omega \epsilon_{1}\right) \rho^{2}}\left\{\frac{\gamma_{1}}{n^{2}}(1\right. \\
& \left.+\gamma_{0} \rho\right) e^{-\gamma_{0} \rho} e^{-\gamma_{1}(z+h)}+\frac{1}{2 \rho^{2}}\left(3+3 \gamma_{1} \rho\right. \\
& \left.\left.+\gamma_{1}{ }^{2} \rho^{2}\right)\left[(z-h) e^{-\gamma_{1} R_{0}}+(z+h) e^{-\gamma_{1} R_{1}}\right]\right\} .
\end{aligned}
$$

A comparison of the two $E_{\phi}$ expressions ((A1) and (A3)) reveals that the difference between them is the unphysical distance $(z+h+\rho)$ term of Wu and King. If we replace $z+h+\rho$ in (A3) by the physical distance $R_{1}$, Wu and King's formula becomes identical to (A1).

A comparison of the two $E_{z}$ expressions ((A2) and (A4)) reveals that $\mathrm{Wu}$ and King's formula does not predict the dip in the interference region because of a sign error. Their results indicate that the sum of the direct and mirror-image waves add to the lateral wave whereas, in reality, the sum subtracts from the lateral wave.

Similar remarks also apply to the HED magnetic field formulas derived by King and Wu [4]. In particular, referring to [4, fig. 2], we see that their $H_{z}$ formula does not predict the interference pattern dip near $\rho \approx 0.08 \mathrm{~m}$.

\section{REFERENCES}

[1] M. B. Kraichman, Handbook of Electromagnetic Propagation in Conducting Media, U.S. Government Printing Office Washington, DC, 1970 , ch. 3.

[2] T. T. Wu and R. W. P. King, "Lateral waves: A new formula and interference patterns," Radio Sci., vol. 17, pp. 521-531, 1982.

[3] _ , "Lateral waves: New formulas for $E_{1 \phi}$ and $E_{1 z}$," Radio Sci., vol. 17, pp. 532-538, 1982.

[4] R. W. P. King and T. T. Wu, "Lateral waves: Formulas for the magnetic field," J. Appl. Phys., vol. 54, pp. 507-514, 1983.

[5] J. R. Wait, "The electromagnetic fields of a horizontal dipole antenna in the presence of a conducting half-space," Can. J. Phys., vol. 39, pp. 1017-1028, 1961.
[6] J. T. Weaver, "The quasi-static field of an electric dipole embedded in a two-layer conducting half-space," Can. J. Phys., vol. 45, pp. 19712002, 1967.

[7] P. R. Bannister, "Quasi-static fields of dipole antennas at the earth's surface," Radio Sci., vol. 1, pp. 1321-1330, 1966.

I [8] P. R. Bannister and W. C. Hart, "Quasi-static fields of dipole antennas below the earth's surface," Naval Underwater Systems Center, New London, CT, Tech. Rep. 870, Apr. 11, 1968; also in P. R. Bannister et al., Quasi-static electromagnetic fields," NUSC Scientific and Engin. Studies, 515 pp. Feb. 1980.

[9] J. R. Wait and L. L. Campbell, "The fields of an electric dipole in a semi-infinite conducting medium," J. Geophys. Res., vol. 58, pp. 21$28,1953$.

[10] infinite conducting medium,'” J. Geophys. Res., vol. 58, pp. 167-178, 1953.

[11] A. K. Sinha and P. K. Bhattacharya, "Vertical magnetic dipole inside a homogeneous earth," Radio Sci., vol. 1, pp. 379-395, 1966.

[12] P. R. Bannister, 'New formulas for HED, HMD, VED, and VMD subsurface-to-subsurface propagation," Naval Underwater Systems Center, New London, CT, Tech. Rep. 6881, Jan. 11, 1984.

[13] K. A. Norton, "The calculation of ground-wave field intensity over a finitely conducting spherical earth,"' Proc. IRE, vol. 29, pp. 623-639, 1941.

[14] K. A. Norton, "The propagation of radio waves over the surface of the earth and in the upper atmosphere, Part I," Proc. IRE, vol. 24, pp. 1367-1387, 1936; _ _ "The propagation of radio waves over the surface of the earth and in the upper atmosphere, Part II," Proc. IRE, vol. 25, pp. 1203-1236, 1937.

[15] R. Li, "The accuracy of Norton's empirical approximations for groundwave attenuation," IEEE Trans. Antennas Propagat., vol. AP-31, pp. 624-628, 1983.

[16] A. Erdélyi, Ed., Tables of Integral Transforms, vol. 2. New York: McGraw-Hill, 1954.

[17] A. C. Fraser-Smith and D. M. Bubenik, "VLF/ELF magnetic fields generated at the sea surface by submerged magnetic dipoles," Radio Sci., vol. 11, pp. 901-913, 1976.

[18] P. R. Bannister, "New formulas that extend Norton's farfield elementary dipole equations to the quasi-nearfield range," Naval Underwater Systems Center, New London, CT, Tech. Rep. 6883, Jan. 11, 1984.

[19]-_, "Extension of finitely conducting earth-image-theory results to any range," Naval Underwater Systems Center, New London, CT, Tech. Rep. 6885, Jan. 11, 1984.

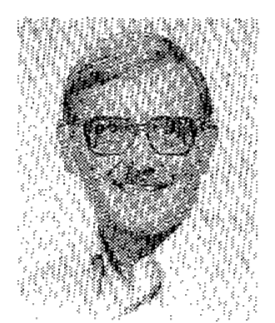

Peter R. Bannister (M'69) was born in Guilford, CT, on August 26, 1938. He received the B.A. and M.S. degree in physics from the University of Connecticut, Storrs, in 1961 and 1965, respectively. Since 1961 he has been with the New London Laboratory, Naval Underwater Systems Center, New London, CT. His main research interests are electromagnetic wave propagation and radiation, extremely low-frequency (ELF) propagation, analytical evaluation of Sommerfeld-type integrals, and extending the use of finitely conducting Earth image theory. He has been assoicated with Project Sanguine/Seafarer/ELF for the past 22 years. From January through September 1982, he occupied the Research Chair in Applied Physics at the Naval Postgraduate School, Monterey, CA. He is also the author of over 150 technical publications.

Mr. Bannister has been awarded over 25 special achievement awards since he has been at the Laboratory, and was the recipient of the New London Laboratory's 1974 Special Achievement Award for excellence in the area of science. 